Espérance de vie en santé : apport conceptuel et pratique de la Classification internationale des déficiences, incapacités, handicaps (CIDIH)

HEALTH EXPECTANCY: CONCEPTUAL AND PRACTICAL CONTRIBUTION OF THE INTERNATIONAL CLASSIFICATION OF IMPAIRMENTS, DISABILITIES AND HANDICAPS (ICIDH) ESPERANZA DE VIDA EN ESTADO SALUDABLE : APORTACION CONCEPTUAL Y PRACTICA DE LA CLASIFICACION INTERNACIONAL DE LAS DEFICIENCIAS, INCAPACIDADES, HANDICAPS (CHDIH)

Pierre Minaire

Volume 20, numéro 2, automne 1991

L’espérance de vie en santé

URI : https://id.erudit.org/iderudit/010085ar

DOI : https://doi.org/10.7202/010085ar

Aller au sommaire du numéro

Éditeur(s)

Association des démographes du Québec

ISSN

0380-1721 (imprimé)

1705-1495 (numérique)

Découvrir la revue

\section{Citer cet article}

Minaire, P. (1991). Espérance de vie en santé : apport conceptuel et pratique de la Classification internationale des déficiences, incapacités, handicaps (CIDIH). Cahiers québécois de démographie, 20(2), 237-251.

https://doi.org/10.7202/010085ar
Résumé de l'article

Le calcul de l'espérance de vie en santé s'appuie sur les mesures de la mortalité et sur celles de l'incapacité. La notion d'incapacité est liée aux définitions modernes de la santé, c'est-à-dire de l'adaptation de la personne ou du groupe à l'environnement. La Classification internationale des déficiences, incapacités, handicaps (CIDIH) permet l'analyse globale des incapacités et des handicaps. Il est possible d'envisager ainsi des définitions communes de la santé et de l'incapacité pour la mise au point d'instruments adaptés aux calculs de l'espérance de vie sans incapacité ou espérance de vie en santé. Il est indispensable que ce travail conceptuel soit effectué au niveau international avant l'établissement de comparaisons entre les données chiffrées d'espérance de vie en santé. De telles comparaisons ne sont licites que si les concepts et les définitions de base sont identiques.
Tous droits réservés @ Association des démographes du Québec, 1991
Ce document est protégé par la loi sur le droit d'auteur. L’utilisation des services d'Érudit (y compris la reproduction) est assujettie à sa politique d'utilisation que vous pouvez consulter en ligne.

https://apropos.erudit.org/fr/usagers/politique-dutilisation/ 
Cahiers québécois de démographie

Vol. 20, no 2, automne 1991, p. 237-251.

\title{
Espérance de vie en santé : apport conceptuel et pratique de la Classification internationale des déficiences, incapacités, handicaps (CIDIH)
}

\author{
Pierre MINAIRE *
}

"L'homme qui a le plus vécu n'est pas celui qui a compté le plus d'années, mais celui qui a le plus senti la vien. Jean-Jacques Rousseau, Émile ou de l'éducation, Bibliothèque de la Pléiade, Livre I. p. 253.

L'espérance de vie en santé et l'espérance de vie sans incapacité s'appuient à la fois sur les mesures de la mortalité et sur celles de la bonne santé et de l'incapacité (Robine et al., 1987; Robine, 1989). Si la mesure de la mortalité ne pose pas de difficultê, il n'en va pas de même pour la mesure de la bonne santé et de l'incapacité. L'hétérogénéité des résultats d'un pays à l'autre ou au sein d'un même pays repose sur des différences de définitions, et met en lumière les faiblesses conceptuelles des définitions choisies. Il faut donc revenir aux données de base sur la santé et l'incapacité pour comprendre l'apport conceptuel et pratique de la Classification internationale des déficiences, incapacités et handicaps (OMS. 1980; CTNERHI/INSERM/ OMS, 1988).

\section{LA SANTÉ}

La santé a d'abord été interprêtée, au XIXe siècle et auparavant, en termes de survie pure. Elle est devenue par la suite l'absence de maladie. Il s'agit là toutefois d'une définition en creux, monofactorielle, qui s'applique particulièrement mal au

Services de rééducation et réadaptation fonctionnelles, et Groupement d'intêrêt public "Effets de l'exercice», Université Jean Monnet, F.4203 Saint-Étienne 2. 
domaine des maladies et symptômes chroniques. En effet, les aspects médicaux et sociaux des affections chroniques sont de distinction difficile : à partir du moment où la notion de durée intervient, au regard tant des symptômes que des traitements, les aspects médicaux de la maladie et leurs consêquences sociales deviennent indissociables.

Plus positive est la définition de l'Organisation mondiale de la santé : la santé est un état de bien-ètre physique, mental, et social complet. C'est aussi la capacité pour l'individu de fonctionner de façon optimale dans son environnement habituel. La santé, c'est en somme l'adaptation à l'environnement lou au milieu).

C'est ici qu'apparait la notion de fonctionnement, c'est-àdire l'accomplissement d'une ou plusieurs fonctions. La fonction, c'est le rôle caractéristique d'une personne dans un ensemble. Rôle, action, utilité sont des caractéristiques sociales liêes à la fonction.

Ainsi, le développement d'un état pathologique durable n'aboutit pas qu'au retrait mécanique d'une personne de l'ensemble auquel elle appartient, mais il affecte la capacité et la performance de la personne en tant que membre participant d'un groupe hautement interdépendant (Fabrega, 1975).

Cette évolution de la définition de la santé vers la capacité et la performance et, partant, vers le bien-être et la qualité de vie perçus et rapportés par la personne explique, en négatif, l'image de l'incapacité considérée comme l'inverse de la bonne santé. L'espérance de vie en bonne santé, c'est avant tout l'espérance de vie sans incapacitê.

\section{L'INCAPACITÉ}

a) La définition de l'incapacité est éminemment variable. Sur un plan international, par exemple, on sait que la prévalence de l'incapacité est liêe au mode de collecte des données et au type de données recueillies (Chamie, 1989). Dans de nombreux endroits, la confusion est entretenue par l'existence de définitions médico-légales de l'incapacité : incapacité permanente partielle française, disability pensions, Disability Act, etc., dont la mise en application a des conséquences parfois considérables pour les budgets sociaux et pour la vie quotidienne des personnes. 
Au delà du débat légal, administratif, et économique, l'incapacité se rapproche de l'inaptitude, qui correspond à des notions plus physiologiques. L'incapacité est le contraire de la capacité, qui, en termes de fonctionnement, doit être soigneusement distinguée de la performance : "je peux monter et descendre les escaliers, mais je ne le fais que rarement car mon environnement habituel est entièrement de plain-pied". L'incapacité n'est donc pas le strict équivalent de la non-performance.

L'incapacité est-elle la restriction d'activité telle qu'elle ressort des enquêtes nationales de santé (Sullivan, 1971; Crimmins et al., 1989) ? Est-ce le taux d'institutionnalisation (Crimmins et al., 1989) ? Mais qu'est-ce qu'une "institution" : ne se définit-elle pas en partie par le degré de dépendance de ceux qui la fréquentent ou y rêsident?

b) La relation entre le degré de l'incapacité et l'état de santé est loin d'être constamment établie. Cette relation doit être de toute manière modulée par l'appréciation de la personne ellemême, et ne peut faire l'objet d'un jugement de valeur absolu de la part de l'observateur. L'évaluation de l'incapacité dépend des réactions humaines de l'observateur et de l'observé, qui sont parfois la même personne (auto-questionnaires). On remarquera toutefois que l'auto-estimation des capacités fonctionnelles peut être tout à fait fiable dans des conditions expérimentales bien déterminées (tableau 1).

TABLEAU 1

Utilisation d'un tapis roulant à $3 \mathrm{~km} / \mathrm{h}$

\begin{tabular}{lcc}
\hline & $\begin{array}{c}\text { Sujets s'estimant } \\
\text { gènés }\end{array}$ & $\begin{array}{c}\text { Sujets s'estimant } \\
\text { non gênés }\end{array}$ \\
\hline Embarquement possible & $34,0 \%$ & $47,6 \%$ \\
Embarquement limité & $48,5 \%$ & $48,1 \%$ \\
Embarquement impossible & $17,5 \%$ & $4,3 \%$ \\
Débarquement possible & $100 \%$ & $100 \%$ \\
Débarquement limité & $46,5 \%$ & $62,5 \%$ \\
Débarquement impossible & $30,9 \%$ & $37,0 \%$ \\
& $16,5 \%$ & $3,9 \%$ \\
\hline
\end{tabular}

Note : Correspondance entre les estimations des sujets testés et les performances observees lors de l'utilisation d'un tapis roulant à $3 \mathrm{~km} /$ heure, village de Saint-Cyr-sur-leRhône. 1984 (Minaire et al., 1987). 
c) L'incapacitẻ recouvre en réalité des chapitres fonctionnels divers. Chaque personne, chaque population a ses préférences et surtout son "profil" en matière d'incapacité (Feinstein et al., 1986). Le même "Score" global d'incapacité recouvre en fait des réalités fonctionnelles différentes: un déficit d'indêpendance physique pour la réalisation des activités quotidiennes, par exemple, pourra être d'origine unique, purement motrice ou cognitive, ou d'origine mixte, cognitive et motrice. Faut-il alors choisir l'espérance de vie sans perte de fonction physique, sans détérioration mentale, ou plutôt sans diminution de performance dans les activités de la vie quotidienne?

d) L'incapacité varie dans le temps : elle s'aggrave souvent, mais elle peut également s'améliorer (Manton, 1988; Chirikos et Nestel, 1985). Les thêrapeutiques rééducatives et réadaptatives sont susceptibles, à tout âge, de stabiliser ou même d'inverser les dêficits fonctionnels dus à la maladie ou au vieillissement, et il faut en tenir compte.

e) L'incapacité dêpend enfin des caractéristiques du milieu et de l'environnement. Le caractère plus ou moins "incapacitant" du milieu influence les calculs d'espérance de vie sans incapacité (Florès et al., 1989; Minaire et al., 1989) (tableau 2). Ces caractéristiques d'environnement ou de milieu peuvent ètre aussi bien architecturales que juridiques, administratives, géographiques ou familiales et humaines.

L'extrême diversité des approches de la bonne santé et de l'incapacité ainsi analysée impose donc une remise en ordre et une clarification conceptuelles permettant de comparer les études et de mettre en place des instruments communs.

\section{LA CLASSIFICATION INTERNATIONALE DES DÉFICIENCES, INCAPACITÉS, HANDICAPS}

La classification internationale des déficiences, incapacités et handicaps a êté publiée récemment (Organisation mondiale de la santé, 1980), à l'issue d'un travail préparatoire qui remonte à 1975 , et selon une résolution de la 29e Assemblée mondiale de la santé de 1976 (réf. WHA 29.5). Une traduction française existe depuis 1982, rééditée sous forme imprimée en 1988 (CTNERHI/INSERM/OMS, 1988).

Il est en effet apparu, peu après la Seconde Guerre mondiale, que les diagnostics médicaux ne pouvaient refléter à eux seuls et avec suffisamment d'exactitude les capacités physiques des 
TABLEAU 2

Espérance de vie sans impossibilité, fermmes de plus de 10 ans, ensemble de la population

\begin{tabular}{lrrrrrrr}
\hline Âge & EV & $\begin{array}{c}\text { EVSI } \\
\text { M35cm }\end{array}$ & $\begin{array}{c}\text { EVSI } \\
\text { M25cm }\end{array}$ & $\begin{array}{c}\text { EVSI } \\
\text { M20cm }\end{array}$ & $\begin{array}{c}\text { EVSI } \\
\text { M15cm }\end{array}$ & $\begin{array}{r}\text { EVSI } \\
\text { Em13 }\end{array}$ & $\begin{array}{r}\text { EVSI } \\
\text { Emt2 }\end{array}$ \\
\hline 10 & 69,7 & 56,5 & 62,0 & 62,6 & 64,7 & 54,0 & 64,7 \\
15 & 64,8 & 51,5 & 57,1 & 57,6 & 59,8 & 49,1 & 59,8 \\
20 & 60,0 & 46,7 & 52,3 & 52,8 & 55,0 & 44,3 & 54,9 \\
25 & 55,1 & 41,9 & 47,3 & 47,9 & 50,0 & 39,5 & 50,0 \\
30 & 50,3 & 37,2 & 42,5 & 43,1 & 45,3 & 34,8 & 45,2 \\
35 & 45,5 & 32,6 & 37,7 & 38,3 & 40,7 & 30,2 & 40,4 \\
40 & 40,7 & 28,1 & 32,9 & 33,5 & 36,0 & 25,7 & 35,6 \\
45 & 36,0 & 23,4 & 28,2 & 28,8 & 31,0 & 21,4 & 30,9 \\
50 & 31,4 & 19,5 & 23,6 & 24,1 & 26,3 & 17,3 & 26,3 \\
55 & 27,0 & 15,5 & 19,2 & 19,6 & 21,8 & 13,8 & 22,0 \\
60 & 22,7 & 11,7 & 14,9 & 15,1 & 17,4 & 10,5 & 17,9 \\
65 & 18,5 & 8,2 & 10,6 & 10,7 & 13,1 & 7,6 & 14,0 \\
70 & 14,5 & 5,2 & 6,7 & 6,7 & 9,0 & 52, & 10,4 \\
75 & 10,9 & 2,8 & 3,5 & 3,5 & 5,6 & 3,4 & 7,4 \\
80 & 7,9 & 0,9 & 1,2 & 1,2 & 3,1 & 2,1 & 5,0 \\
85 & 5,5 & 0,0 & 0,0 & 0,0 & 1,4 & 1,1 & 3,3 \\
\hline
\end{tabular}

Note : Résultats du calcul d'espérance de vie sans impossibilité de passage de marches de hauteur variable. et sans impossibilité d'utilisation d'un tapis roulant à deux vitesses chez les femmes de plus de dix ans, dans l'ensemble de la population de Sainit-Cyr-sur-Je-Rhōne. 1984 (Florès et al., 1989). Signification des sigles :

EV : Espérance de vie (années).

EVSI M35cm : Espérance de vie sans impossibilité, passage de marches de $35 \mathrm{~cm}$ sans appui.

EVSI M25cm : Espérance de vie sans impossibilité. passage de marches de $25 \mathrm{~cm}$ sans appui.

EVSI M20cm : Espérance de vie sans impossibilité, passage de marches de $20 \mathrm{~cm}$ sans appui.
EVSI M $15 \mathrm{~cm}$ : Espérance de vie sans impossibilité, passage de marches de $15 \mathrm{~cm}$ sans appui.

EVSI Emt3 : Espérance de vie sans impossibilité, utilisation tapis roulant $3 \mathrm{~km} / \mathrm{h}$. EVSI Emt2 : Espérance de vie sans impossibilité, utilisation tapis roulant $2 \mathrm{~km} / \mathrm{h}$.

personnes atteintes d'affections chroniques ou des personnes âgées. L'évaluation de l'incapacité était donc un complément nécessaire du diagnostic médical (Moskowitz et McCann, 1957). Cette réflexion a été formalisée par Wood entre 1976 et 1980 , selon la ligne de réflexion suivante : une maladie, un traumatisme, une altération génétique, entraînent chez une personne donnée l'apparition d'une anomalie. Cette anomalie est perçue par la personne, ou observée par un tiers (prise de conscience ou objectivation). Des conséquences fonctionnelles en découlent, qui réduisent les performances ou altèrent le comportement de la personne. La prise de conscience ou la modification de performance ou de comportement peuvent placer la personne 
en situation de désavantage dans la vie quotidienne, ce qui l'empêche de tenir le rôle qu'elle-même ou la sociêté attendent d'elle.

Ce raisonnement simple, structuré à une époque où apparait une demande accrue d'étude des causes ou des prévalences et d'évaluation des services dans le domaine de l'incapacité, a conduit au développement d'un cadre conceptuel d'ensemble incorporant les sphères diverses, bien qu'apparentées, du domaine de la santé définie au sens large. Ce cadre comporte trois dimensions et se rattache au modèle biomédical classique selon le schéma :

Maladie

$\left.\begin{array}{l}\text { (ou traumatisme, } \\ \text { ou anomalie) }\end{array}\right\} \rightarrow$ dèfícience $\rightarrow$ incapacité $\rightarrow$ handicap

Ce schéma est celui d'un processus linéaire de handicap lau sens du mot anglais "disablement") dont chaque élément peut faire l'objet d'une analyse séparée.

1. La maladie ne se définit pas qu'en variables anatomiques, biochimiques ou physiologiques, mais s'associe aussi à des facteurs physiques, culturels ou sociaux, comme cela a déjà été mentionné ci-dessus. Toutefois, certains éléments tels que diagnostics, symptômes ou paramètres biologiques ont une valeur prospective pour l'analyse du processus global de handicap, ne serait-ce qu'à titre d'élêments clés de l'introduction de la personne dans le processus. Des diagnostics tels que les accidents vasculaires cérébraux, les fractures du col du fémur, les rhumatismes inflammatoires, ou des symptomes tels que la dyspnée ou la claudication intermittente à la marche, par exemple, sont associés à une probabilité élevée de conséquences fonctionnelles et de réduction de performances. L'incidence et la prévalence de ces êléments cliniques ne sont donc pas dénuées d'intérêt pour l'êtude de l'incapacité.

2. "Dans le domaine de la santé, la déficience correspond à toute perte de substance ou altération d'une fonction ou d'une structure psychologique, physiologique, ou anatomique" (CTNERHI/INSERM/OMS, 1988). La déficience peut être temporaire ou définitive. Elle représente l'extêriorisation d'un processus pathologique sous-jacent. Elle est le reflet des troubles manifestés au niveau de l'organe. Sa reconnaissance et sa mesure ne posent pas de problème à l'aide des bilans cliniques, biologiques ou physiologiques, des explorations fonctionnelles ou des évaluations habituelles des services cliniques. 
3. "L'incapacité correspond, dans le domaine de la santé, et à la suite d'une déficience, à toute réduction partielle ou totale de la capacité d'accomplir une activité d'une façon, ou dans les limites, considérées comme normales pour un être humain" (CTNERHI/INSERM/OMS, 1988). L'incapacité peut être temporaire ou permanente, rêversible ou irréversible, progressive ou soudaine. Elle représente l'objectivation d'une déficience, et reflète les perturbations au niveau de la personne elle-même.

La dimension d'incapacité concerne les activités et comportements composites généralement considérês comme des éléments essentiels de la vie quotidienne: communiquer, se dẻplacer, se nourrir, se comporter, etc. L'appréciation et lla mesure de l'incapacité sont donc centrées sur les activités de la vie quotidienne. Des échelles et des grilles nombreuses permettent d'analyser l'incapacité, sous la forme d'un score global reproductible, et d'un profil individuel ou par groupes basé sur l'ensemble des rubriques incluses dans l'instrument de mesure (Mahoney et Barthel, 1965; Katz et al., 1963; Hamilton et al., 1987; Gardent et al., 1988).

4. "Le handicap, ou désavantage social d'une personne, est, dans le domaine de la santé, le préjudice qui résulte de sa déficience ou de son incapacité, et qui limite ou interdit l'accomplissement d'un rôle considéré comme normal compte tenu de l'âge, du sexe, et des facteurs socio-culturels" (CTNERHI/ INSERM/OMS, 1988). La CIDIH comporte six chapitres de désavantages possibles: orientation, indépendance physique, mobilité, intégration sociale, indépendance économique, activité professionnelle. Ces six chapitres correspondent aux situations dans lesquelles les personnes présentant une incapacité peuvent se trouver en difficulté, ce qui les place en position de désavantage par rapport à leurs pairs du point de vue des normes sociales. Des questionnaires permettent d'analyser les performances de la personne, c'est-à-dire la réalisation des activités par choix de la personne ou par nécessité sociale reconnue et acceptée. Néanmoins, peu d'études concernent encore cette dimension fondamentale du processus de handicap (Colvez et Robine, 1986; Whiteneck, 1987; Badley et al., 1990), qui est pourtant la plus prometteuse sur le plan opérationnel.

\section{APPROCHE SYNTHÉTIQUE DE LA CIDIH}

La CIDIH, dans sa partie conceptuelle autant que dans sa partie classificatoire, est un outil d'analyse particulièrement 
intéressant. En effet, le processus global ainsi dêcrit correspond à trois dimensions bien définies qui recouvrent chacune des plans différents d'expérience. Ces plans sont néanmoins indissociables pour l'analyse globale de l'incapacitê et du handicap. Le tableau 3 en résume quelques exemples.

TABLEAU 3

Exemple d'analyse globale de la déficience, de l'incapacité et du handicap par grandes catégories

\begin{tabular}{|c|c|c|}
\hline Déficience & Incapacité & Handicap \\
\hline Langage & Parler & Occupation \\
\hline Audition & Écouter & Orientation \\
\hline Vision & Voir & Mobilité \\
\hline Locomotion & $\begin{array}{l}\text { S'habiller } \\
\text { Se nourrir } \\
\text { Marcher ou } \\
\text { se déplacer }\end{array}$ & $\begin{array}{c}\text { Indépendance } \\
\text { Indépendance physique } \\
\text { Mobilité }\end{array}$ \\
\hline $\begin{array}{c}\text { Psychologique } \\
\ldots .\end{array}$ & $\begin{array}{c}\text { Comportement } \\
\ldots\end{array}$ & $\begin{array}{c}\text { Intègration sociale } \\
\ldots\end{array}$ \\
\hline
\end{tabular}

Il est possible également d'effectuer à l'aide de la CIDIH une analyse complète des conséquences des maladies, traumatismes ou anomalies. Des exemples non exhaustifs en sont donnés au tableau 4. Chaque cas, chaque échantillon de population peut faire l'objet, dans le domaine de la santé, d'analyses similaires.

Le plus grand avantage de la CIDIH réside dans l'ouverture de ses plans d'expẻrience à tous les facteurs déterminants de l'incapacité, tant médicaux que sociaux ou techniques. Cela permet un langage et une lecture communs, non monolithiques, "déglobalisants", de l'incapacité et du handicap, car non exclusivement médicaux (Zucman, 1982). Ce langage commun à divers intervenants est aussi un remarquable outil d'enseignement.

La CIDIH permet également de faire une analyse dynamique du processus d'incapacité et de handicap, au delà des concepts surannés et statiques d'invalidité, de dépendance, ou de vieillesse, en évitant la partition habituelle entre les conséquences médicales et sociales de la maladie. La CIDIH et ses dimensions sont utilisables comme base d'évaluation individuelle ou de populations (Colvez et Robine, 1986; Minaire et al., 1987; INS, 
TABLEAU 4

Exemple d'analyse de la déficience, de l'incapacité et du handicap en relation avec certains diagnostics

\begin{tabular}{|c|c|c|c|}
\hline Diagnostic & Déficience & Incapacité & Handicap \\
\hline $\begin{array}{l}\text { Accident } \\
\text { vasculaire } \\
\text { cérébral }\end{array}$ & Hémiplégie & $\begin{array}{l}\text { Difficulté de : } \\
\text { - communication } \\
\text { - réalisation } \\
\text { des soins } \\
\text { personnels } \\
\text { - marche } \\
\text { - utilisation } \\
\text { du corps } \\
\text { - } \ldots\end{array}$ & $\begin{array}{l}\text { Réduction de : } \\
\text { - mobilité } \\
\text { - indépendance } \\
\quad \text { physique } \\
\text { - intégration } \\
\quad \text { sociale } \\
\text { Difficultês } \\
\text { économiques } \\
\ldots\end{array}$ \\
\hline $\begin{array}{l}\text { Arthrose } \\
\text { de hanche }\end{array}$ & $\begin{array}{l}\text { Raideur et } \\
\text { douleur de } \\
\text { la hanche }\end{array}$ & $\begin{array}{l}\text { Difficulté de : } \\
\text { - marche } \\
\text { - réalisation } \\
\text { des soins } \\
\text { personnels }\end{array}$ & $\begin{array}{l}\text { Mobilité réduite } \\
\text { Indépendance } \\
\text { physique réduite } \\
\text { Intégration sociale } \\
\text { perturbée }\end{array}$ \\
\hline $\begin{array}{l}\text { Antécédents } \\
\text { diaccouchements } \\
\text { difficiles } \\
\text { Ou syndrome } \\
\text { frontal } \\
\text { Ou déficit hormonal }\end{array}$ & $\begin{array}{l}\text { Sphincter } \\
\text { urétral } \\
\text { incompétent }\end{array}$ & $\begin{array}{l}\text { Pertes } \\
\text { involontaires } \\
\text { d'urine }\end{array}$ & $\begin{array}{l}\text { Mobilité réduite } \\
\text { Intégration sociale } \\
\text { perturbée }\end{array}$ \\
\hline Diabète & $\begin{array}{l}\text { Acuité visuelle } \\
\text { réduite } \\
\text { Paralysie } \\
\text { Assujettissement } \\
\text { au traitement } \\
\text { Sphincters } \\
\text { hypotoniques } \\
\text {... }\end{array}$ & $\begin{array}{l}\text { Vision perturbée } \\
\text { Difficulté de : } \\
\text { - marche } \\
\text { - réalisation } \\
\text { des soins } \\
\text { personncls } \\
\text { Pertes d'urine }\end{array}$ & $\begin{array}{l}\text { Mobilité réduite } \\
\text { Intégration sociale } \\
\text { perturbée } \\
\text { Difficultés } \\
\text { économiques et } \\
\text { professionnelles } \\
\ldots\end{array}$ \\
\hline
\end{tabular}

1987; MacAndrew et Hanley, 1988; Cucherousset, 1991) (figure 1). En santé publique, la CIDIH permet d'orienter la prévention sur les trois dimensions du processus, et non pas seulement sur la prévention primaire classique des maladies potentiellement handicapantes. Les distinctions ainsi faites en matière de prêvention sont particulièrement utiles dans le dialogue entre décideurs, professionnels de la santé et usagers. et permettent de cibler le choix des objectifs de prévention. La même remarque peut être faite en ce qui concerne la planification des services. 


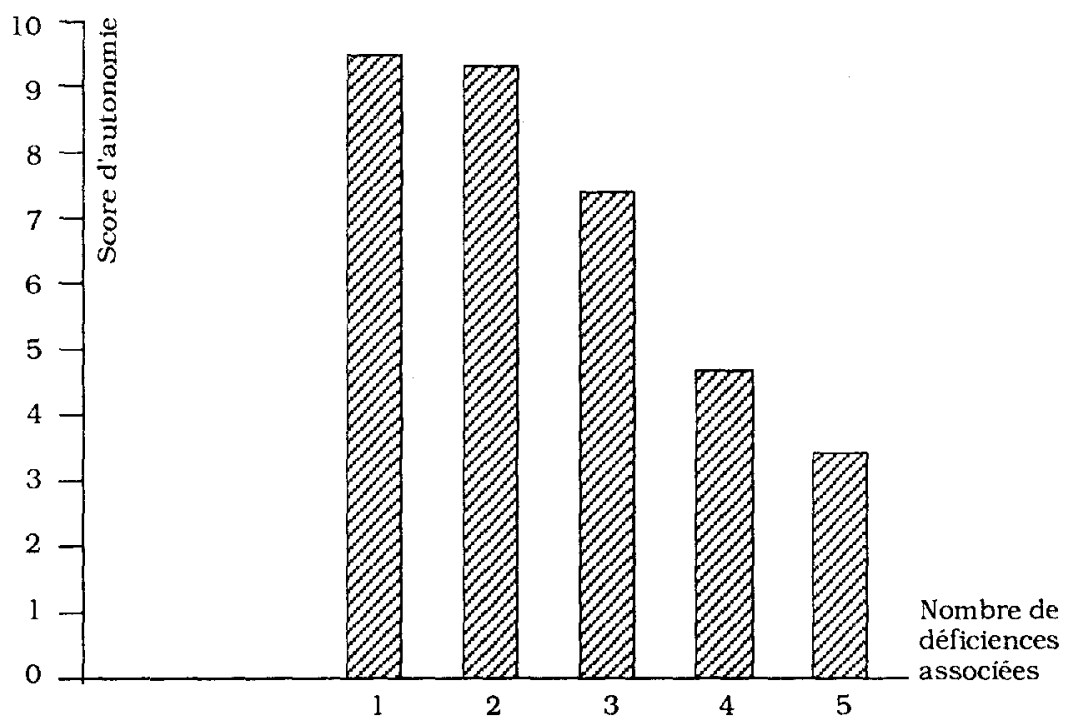

Source : Cucherousset, 1991.

Figure 1 - SCORE D'AUTONOMIE EN RELATION AVEC LE NOMBRE DE DÉFICIENCES ASSOCIÉES, ENGUÊTE SUR 1845 ENFANTS HANDICAPESS EN INSTITUTION DANS LE DÉPARTEMENT DE LA LOIRE, EFFECTUEE SELON LES DIMENSIONS DE LA CIDIH

La CIDIH offre donc un support épidémiologique différent et oriente l'information et la collecte des données utiles sur l'ensemble des problèmes posés par les personnes en état d'incapacité et de handicap. Il reste à y associer une évaluation de l'environnement non seulement physique, mais aussi social, juridique, familial et économique des personnes. Il convient en effet de considérer aussi les personnes en situation : ce sont bien les situations de la vie quotidienne, telles qu'on peut les objectiver et telles qu'elles sont perçues par les personnes en désavantage ou par leur entourage, qui, confrontêes à l'incapacitê, réduisent ou gênent aussi la performance de la personne (Minaire, 1983; Fougeyrollas et al., 1989).

\section{CIDIH ET INDICATEURS D'ESPÉRANCE DE VIE EN SANTÉ}

La CIDIH constitue ainsi un cadre conceptuel clair et substantiel pour l'analyse transdisciplinaire de ce qu'est la vie en santé, ou sans incapacité. Sa structure tridimensionnelle peut servir de modèle pour les enquêtes de prévalence de l'incapa- 
cité. Il est encore difficile de se prononcer sur son utilité dans les études de l'incidence des conditions chroniques sources d'incapacitê. Pour cela, la linéarité de la chaîne de causalité entre les trois dimensions de la déficience, de l'incapacité et du handicap doit être démontrée, et les types de transitions interdimensionnelles doivent être définis plus précisément. L'incapacité et le handicap sont les deux dimensions importantes à considérer pour le calcul de l'espérance de vie en santé. Diagnostic et déficience sont en effet trop liês au concept biomédical classique. Si l'incapacité peut être considérée comme un prédicteur de l'ajustement à la vie sociale, le handicap ou désavantage est un composant de cet ajustement.

Les indicateurs les plus fiables de l'incapacité sont actuellement la locomotion (ou faculté de se mouvoir) et les activités de la vie quotidienne (habillage, alimentation, hygiène corporelle, contrôle sphinctérien). Les indicateurs correspondants du handicap et du désavantage vécu sont, en regard, la mobilité, c'est-à-dire la faculté de se déplacer aux moments et sur les itinéraires choisis, et l'indépendance physique, c'est-à-dire la possibilité d'accomplir seul ou aidé les activités de la vie quotidienne. Les éléments de mobilité et d'indépendance physique recouvrent, mais en partie seulement, le concept de qualité de vie en relation avec la santé (Minaire, 1990).

Le choix entre l'incapacité et le handicap ou désavantage ou entre la capacité et la performance réelle pour l'incorporation dans les calculs d'espérance de vie en santé n'est pas simple. Il est plus facile d'obtenir des informations sur l'incapacité, car les questionnaires dans ce domaine ne nécessitent pas de jugements complexes. Il existe même des instruments modernes (Hamilton et al., 1987; Martin et al., 1988) pour l'êtablissement d'un score d'incapacité valide, fiable, sensible et reproductible.

Néanmoins, la dimension du handicap ou désavantage vécu devrait également donner lieu dans le futur à des développements de questionnaires et à des études spécifiques. De toute êvidence, incapacité et handicap sont deux dimensions très complémentaires et très liées, bien que de définition différente. On peut ainsi postuler que :

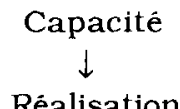

Réalisation des activitès de la vie quotidienne

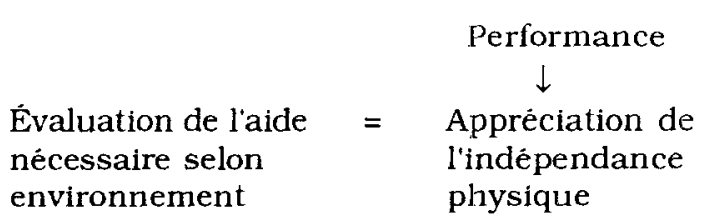

Performance

Appréciation de physique 
ou encore que :

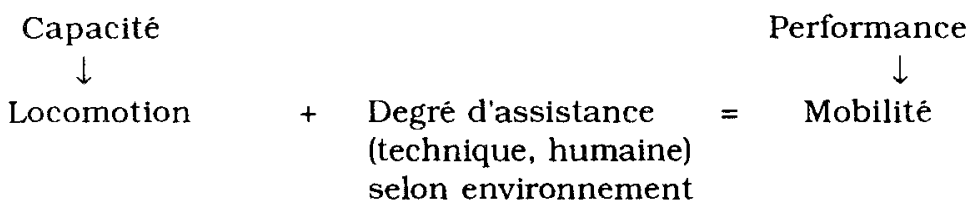

En conclusion, il apparaît que, pour les calculs d'espérance de vie en santé, le choix des indicateurs ou le type d'aptitudes étudiées sont loin d'être neutres et peuvent influencer significativement les données. Il semble donc approprié de recommander :

- de choisir des indicateurs et des instruments explorant les champs d'incapacité et de handicap ou désavantage selon les définitions de la CIDIH,

- de prendre en compte les facteurs culturels et environnementaux et de collecter des données concernant plus les situations que les individus,

- de choisir en première intention, pour les calculs, les activités quotidiennes et la locomotion (incapacités) et l'indépendance physique et la mobilite (handicaps), qui sont pour le moment les domaines d'investigation les plus fiables et les plus transculturels,

- de classer les études disponibles d'espérance de vie en santé selon les dimensions de la CIDIH auxquelles elles se réfèrent, que la rêférence soit explicite ou non.

La CIDIH et ses concepts permettent tout à fait la mise en oeuvre de ces recommandations, et peuvent contribuer à la mise au point d'autres instruments d'analyse de complexité variable selon les rubriques que l'on désire y inclure.

\section{RÉFÉRENCES BIBLIOGRAPHIGUES}

BADLEY, E. M., A. TENNANT et P. H. N. WOOD, 1990. "The Assessment of Physical Independence Handicap: Experience in a Community Disablement Survey". International Disability Studies, 12, 47-53.

CHAMIE, M., 1989. "Survey Design Strategies for the Study of Disability". World Health Statistics Quarterly, 42, 3, 122-140.

CHIRIKOS, T. N., et G. NESTEL, 1985. "Longitudinal Analysis of Functional Disabilities in Older Men". Journal of Gerontology, 40, 426-433. 
COLVEZ, A., et J.-M. ROBINE, 1986. „Problems Encountered in Using the Concepts of Impairment, Disability, and Handicap in a Health Assessment Survey of the Elderly in Upper Normandy". International Rehabilitation Medicine, 8, 18-22.

CRIMMINS, E. M., Y. SAITO et D. INGEGNERI, 1989. "Changes in LifeExpectancy and Disability-Free Life Expectancy in the United States». Population and Development Review, 15, 2, 235-267.

CTNERHI/INSERM/OMS, 1988. Classification internationale des handicaps : déficiences, incapacités, handicaps. Un manuel de classification des conséquences des maladies. Paris, Diffusion Presses universitaires de France, 203 p.

CUCHEROUSSET, C., 1991. Les enfants et adolescents handicapés en établissements : étude épidémiologique portant sur 1845 dossiers dans un département de 750 ooo habitants. Thèse, Faculté de médecine Jacques Lisfranc, Université Jean Monnet, F-42023 Saint-Étienne 2, no 05, 115 p.

FABREGA. H., 1975. "The Need for an Ethnomedical Science. Science, 189, 969-975.

FEINSTEIN, A. R., B. R. JOSEPHY et C. K. WELLS, 1986. "Scientific and Clinical Problems in Indexes of Functional Disabilities". Annals of Internal Medicine, 105, 413-420.

FLORĖ, J. L., P. MINAIRE, J. CHERPIN et D. WEBER, 1989. Mesure de l'incapacité et caractéristiques du milieu : influence sur le calcul de l'espérance de vie sans incapacité. In: Première rencontre de travail REVES, Réseau international de recherche sur l'espérance de vie en santé, Québec, septembre. Sous presse : Paris, Éditions INSERM, collection "Analyses et prospective".

FOUGEYROLLAS, P., G. ST-MICHEL et M. BLOUIN, 1989. "Consultation: proposition d'une révision du 3ème niveau de la $\mathrm{CIDIH}$ : le handicap". In Réseau international CIDIH, vol. 2, no 1, 29 p.

GARDENT, H., J. SPINGA et A. BOUNOT, 1988. Dépendance des personnes âgées et charge en soins. Expérimentation simultanée de six grilles de dépendance. Paris, CTNERHI, Diffusion Presses universitaires de France, 396 p.

HAMILTON, B. B., C. V. GRANGER, F. S. SHERWIN, M. ZIELEZNY et J. S. TASHMAN, 1987. "A Uniform Data System for Medical Rehabilitation". In M. J. FUHRER, èd. Rehabilitation Outcomes. Baltimore, Brookes, 276 p., 137-147.

INS (INSTITUT NATIONAL DE STATISTIQUE), 1987. Enquête sur les déficiences, les incapacités et les désavantages. Madrid, Institut national de statistique, vol. I et II.

KATZ, S., C. A. AKPOM, J. A. PAPSIDERO et S. T. WEISS, 1973. "Measuring the Health Status of Populations". In R. L. BERG, éd. Health Status Indexes. Chicago. Hospital Research and Education Trust, 39-52.

MacANDREW, L., et J. HANLEY, 1988. Survey of the Younger Disabled in the Community in Lothian. Edimbourg, Rehabilitation Studies Unit, $67 \mathrm{p}$. 
MAHONEY, F. I., et D. W. BARTHEL, 1965. «Functional Evaluation: The Barthel Index". Maryland State Medicine Journal, 14, 61-65.

MANTON, K. G., 1988. "A Longitudinal Study of Functional Change and Mortality in the United States*. Journal of Gerontology: Social Sciences, 43, 5, S153-S161.

MARTIN, J., H. MELTZER et D. ELLIOT, 1988. The Prevalence of Disability among Adults. OPCS Surveys of Disability in Great Britain, Report 1. OPCS Social Survey Division, Londres, Her Majesty's Stationery Office.

MINAIRE, P., 1983. "Le handicap en porte-à-faux". Prospective et santé, 26, 29-46.

MINAIRE, P., 1990. Theoretical Models of the Disability Process. In 3rd Work-group Meeting REVES, International Research Network for Interpretation of Observed Values of Healthy Life Expectancy. Durham, NC, USA, décembre, Paper No. 43.

MINAIRE, P., J. CHERPIN, J. L. FLORES et D. WEBER, 1989. "La mesure du handicap dans la communauté : une micro-enquête dans un village français». World Health Statistics Quarterly/Rapport trimestriel des statistiques sanitaires mondiales, 42, 167-176.

MINAIRE, P.. J. L. FLORÈS et J. CHERPIN, 1987. *The Functional Aptitude of a Natural Sample of Population: A New Approach to Handicap». International Disability Studies, 9, 65-68.

MOSKOWITZ, E., et C. B. McCANN, 1957. "Classification of Disability in Chronically Ill and Aging". Journal of Chronic Disease, 5, 342346.

OMS/WHO (Organisation mondiale de la santé / World Health Organization). 1980. International Classification of Impairments, Disabilities and Handicaps. A Manual of Classification Relating to the Consequences of Disease. Genève, OMS, $201 \mathrm{p}$.

ROBINE, J.-M., 1989. "Estimation de la valeur de l'espérance de vie sans incapacité (EVSI) pour les pays occidentaux au cours de la dernière décennien. World Health Statistics Quarterly/Rapport trimestriel des statistiques sanitaires mondiales, 42, 3, 141-150.

ROBINE, J.-M., N. BROUARD et A. COLVEZ, 1987. "Les indicateurs d'espérance de vie sans incapacité (EVSI) : des indicateurs globaux de l'état de santé des populations". Revue d'épidémiologie et de santé publique, 35, 206-224.

SULLIVAN, D. F., 1971. "A Single Index of Mortality and Morbidity". HSMHA Health Reports, 86, 4, 347-354.

WHITENECK, G. G., 1987. "Outcome Analysis in Spinal Cord Injury Rehabilitation". In M. J. FUHRER, èd. Rehabilitation Outcomes, Analysis and Measurement. Baltimore, Brookes, 276 p., 221-234.

ZUCMAN, E., 1982. L'identification individualisée pluri-dimensionnelle de la situation des personnes handicapées ou en difficulté d'adaptation en France. Adaptation de la classification OMS des déficiences, incapacités, handicaps, aux besoins de la recherche et de la pratique d'action sociale. Handicaps et inadaptations. Les Cahiers du CTNERHI, 20, 422-448. 


\section{RÉSUMÉ - SUMMARY — RESUMEN}

\section{MINAIRE PieTre - ESPÉRANCE DE VIE EN SANTÉ : APPORT CONCEPTUEL ET PRATIGUE DE LA CLASSIFICATION INTERNATIONALE DES DEFI- CIENCES, INCAPACITÉS, HANDICAPS (CIDIH)}

Le calcul de l'espérance de vie en santé s'appuie sur les mesures de la mortalité et sur celles de l'incapacité. La notion d'incapacité est liée aux définitions modernes de la santé, c'est-à-dire de l'adaptation de la personne ou du groupe à l'environnement. La Classification internationale des déficiences, incapacités, handicaps (CIDIH) permet l'analyse globale des incapacités et des handicaps. Il est possible d'envisager ainsi des définitions communes de la santé et de l'incapacité pour la mise au point d'instruments adaptés aux calculs de l'espérance de vie sans incapacité ou espérance de vie en santé. Il est indispersable que ce travail conceptuel soit effectué au niveau international avant l'établis. sement de comparaisons entre les données chiffrées d'espérance de vie en santé. De telles comparaisons ne sont licites que si les concepts et les définitions de base sont identiques.

\section{MINAIRE Pierre - HEALTH EXPECTANCY: CONCEPTUAL AND PRACTICAL CONTRIBUTION OF THE INTERNATIONAL CLASSIFICATION OF IMPAIRMENTS, DISABILITIES AND HANDICAPS (ICIDH)}

Calculation of health expectancy is based on measurements of both mortality and disability. The notion of disability is linked to modern definitions of health, i.e. how persons or groups adapt to their environment. The International Classification of Impairments, Disabilities and Handicaps (ICIDH) provides a means to make an overall analysis of disabilities and handicaps, thus allowing us to envisage common definitions of health and disability to be used in developing instruments suitable for calculating disability-free life expectancy or health expectancy. It is essential that this development work be carried out on an international scale before going on to make comparisons between statistical data on health expectancy. since such comparisons will only be valid if the basic concepts and definitions used are the same.

MINAIRE Pierre - ESPERANZA DE VIDA EN ESTADO SALUDABLE : APORTACION CONCEPTUAL Y PRACTICA DE LA CLASIFICACION INTERNA. CIONAL DE LAS DEFICIENCIAS, INCAPACIDADES, HANDICAPS (CHDIH)

El cálculo de la esperanza de vida en estado saludable se basa en las medidas de la mortalidad asi como en las de la incapacidad. La noción de incapacidad está vinculada a las definiciones modernas de salud, o sea la adaptación de la persona o del grupo al medio ambiente. La Clasificación Internacional de las Deficiencias, Incapacidades, Handicaps (CIDIH) permite efectuar el análisis global de las incapacidades y de los handicaps. Así, definiciones comunes de la salud y de la incapacidad pueden ayudar a implementar instrumentos que se adapten a los cálculos de la esperanza de vida sin incapacidad, o esperanza de vida en estado saludable. Es indispensable que este trabajo conceptual se lleve a cabo a nivel internacional, antes de establecer comparaciones entre los datos cifrados de esperanza de vida en estado saludable. Dichas comparaciones no pueden ser lícitas más que si resultan identicos los conceptos y las definiciones básicas. 